OPEN ACCESS

Edited by:

Sanni Yaya

University of Ottawa, Canada

Reviewed by:

Renee Bolijn,

Academic Medical

Center, Netherlands

Julia Smith,

Simon Fraser University, Canada

${ }^{*}$ Correspondence:

Amy Vassallo

avassallo@georgeinstitute.org.au

Specialty section:

This article was submitted to

Sex and Gender Differences in

Disease,

a section of the journal

Frontiers in Global Women's Health

Received: 19 August 2021

Accepted: 08 October 2021

Published: 01 November 2021

Citation:

Vassallo A, Shajahan S, Harris K Hallam L, Hockham C, Womersley $K$, Woodward M and Sheel M (2021) Sex and Gender in COVID-19 Vaccine Research: Substantial Evidence Gaps

Remain.

Front. Glob. Womens Health

2:761511.

doi: 10.3389/fgwh.2021.76151

\section{Sex and Gender in COVID-19 Vaccine Research: Substantial Evidence Gaps Remain}

\author{
Amy Vassallo ${ }^{1 *}$, Sultana Shajahan ${ }^{1}$, Katie Harris ${ }^{1}$, Laura Hallam ${ }^{1}$, Carinna Hockham ${ }^{2}$, \\ Kate Womersley ${ }^{2,3}$, Mark Woodward ${ }^{1,2}$ and Meru Sheel ${ }^{4}$ \\ ${ }^{1}$ The George Institute for Global Health, University of New South Wales, Sydney, NSW, Australia, ${ }^{2}$ The George Institute for \\ Global Health, School of Public Health, Imperial College London, London, United Kingdom, ${ }^{3}$ University of Edinburgh, NHS \\ Lothian, Edinburgh, United Kingdom, ${ }^{4}$ National Centre for Epidemiology and Population Health, Research School of \\ Population Health, ANU College of Health and Medicine, Australian National University, Canberra, ACT, Australia
}

Since the start of the COVID-19 pandemic there has been a global call for sex/gender-disaggregated data to be made available, which has uncovered important findings about COVID-19 testing, incidence, severity, hospitalisations, and deaths. This mini review scopes the evidence base for efficacy, effectiveness, and safety of COVID19 vaccines from both experimental and observational research, and asks whether (1) women and men were equally recruited and represented in vaccine research, (2) the outcomes of studies were presented or analysed by sex and/or gender, and (3) there is evidence of sex and/or gender differences in outcomes. Following a PubMed search, 41 articles were eligible for inclusion, including seven randomised controlled trials (RCTs), 11 cohort studies, eight cross-sectional surveys, eight routine surveillance studies, and seven case series. Overall, the RCTs contained equal representation of women and men; however, the observational studies contained a higher percentage of women. Of 10 studies with efficacy data, only three (30\%) presented sex/gender-disaggregated results. Safety data was included in 35 studies and only 12 (34\%) of these presented data by sex/gender. For those that did present disaggregated data, overall, the majority of participants reporting adverse events were women. There is a paucity of reporting and analysis of COVID-19 vaccine data by sex/gender. Research should be designed in a gender-sensitive way to present and, where possible analyse, data by sex/gender to ensure that there is a robust and specific evidence base of efficacy and safety data to assist in building public confidence and promote high vaccine coverage.

Keywords: COVID-19, vaccine, immunisation, evidence, gender equality, safety, gender

\section{INTRODUCTION}

The important influence of sex and gender on health has come to the forefront during the COVID19 pandemic. Globally, shared sex-disaggregated data has led to important understanding about COVID-19 testing, incidence, severity, hospitalisations, and deaths (1-4). For example, while the proportion of COVID cases in women and men are roughly equal, men have around three times the odds of intensive care admission and a 40\% higher odds of dying from COVID-19 than women (4). Known biological differences in adaptive and innate immune responses between sexes explain some of these observed differences (4). Socio-cultural gender constructs also influence 
these outcomes through differing exposures to the disease (such as high occupational exposure in frontline healthcare workers, who are predominantly women), risk factors for severe disease (such as higher smoking rates in men), existence of comorbidities, and engagement with healthcare services for prevention, detection, and treatment (typically lower in men) (5-8).

Sex and gender are also important factors in understanding immunisation, including vaccine delivery, efficacy, and frequency and severity of adverse reactions (6). Sex and gender differences in immunisation outcomes have been observed across age groups for other vaccine preventable diseases, with women typically developing higher antibody responses, and reporting more local and systemic adverse reactions, compared with men (9). These differences have been observed in response to vaccines using different technologies, including the CalmetteGuerin vaccine, measles, mumps and rubella, yellow fever, and influenza vaccines (10). Several biological mechanisms have been proposed, including immunological, hormonal, genetic, and microbiota differences between females and males $(10,11)$.

Developing an effective vaccine against COVID-19 has been a global research priority, with several different vaccines administered on a large scale across the globe in 2020 and 2021 as part of national immunisation programs. This shift from experimental to observational (including routine surveillance) research provides valuable acceptability, effectiveness, and real world safety data (12). Effective, efficient, equitable, and publicly acceptable immunisation programs are needed for control of the COVID-19 pandemic globally. These programs need to be rooted in sex- and gender-sensitive evidence. The aim of this mini review is to scope the evidence base for efficacy, effectiveness and safety of COVID-19 vaccines, and whether (1) women and men were equally recruited and represented in each vaccine's research, (2) the outcomes of studies were presented or analysed by sex and/or gender, and (3) there is evidence of sex and/or gender differences in outcomes.

\section{METHODS}

\section{Search Strategy and Selection Criteria}

We searched PubMed for peer-reviewed literature on the efficacy, effectiveness, and/or safety of COVID-19 vaccines included in the COVID-19 Vaccines Global Access (COVAX) portfolio as of 6 May 2021: Pfizer/BioNTech (BNT162b2), Oxford/AstraZeneca (AZD1222), Novavax (NVX-CoV2373), Covovax (NVX-CoV2373), Johnson\&Johnson (Ad26.COV2.S), Sanofi/GSK (VAT00002), and Moderna (mRNA-1273) $(13,14)$. Search terms relating to vaccine name, SARS-CoV-2, COVID19, COVID-19 vaccines, phase 3 and 4 clinical trials, efficacy, effectiveness and mass vaccination, and relevant synonyms of these, were used (see Appendix 1 for detailed search strategy). To identify articles containing safety data, additional search terms relating to adverse effects, safety monitoring, safety profile, and appropriate synonyms, were used. Searches were conducted using standard keywords as well as MeSH terms.

Eligible studies included phase $2 / 3$ or 3 randomised controlled trials (RCTs) (experimental studies) and post-market observational studies including cohort studies, cross-sectional studies, routine surveillance reports, and case series. Animal studies, phase 1 and/or 2 RCTs and case studies were excluded. All article types that presented original data were included, including research articles, editorials, responses, and letters to the editor. Immunogenicity data were excluded, however any relevant safety outcome data from these studies were included. In case of duplicate publications containing the same data, the report with the greatest amount of data or the one published first was included.

\section{Data Extraction and Analysis}

Title/abstract screening, full-text review and data extraction were conducted in duplicate by AV (all papers) and SS, KH, LH, and $\mathrm{CH}$. Any disagreements between reviewers were resolved by discussion. Data were extracted for study title, author, date of publication, vaccine name(s), study design, population subgroup (e.g. healthcare workers, people with pre-existing conditions), percentage of women and men participants, vaccine efficacy and effectiveness (as defined by study authors), and the percentage of women and men who presented with adverse events (as defined by study authors). Results data for up to 12 adverse events per article were extracted. Care was taken not to report the occurrence of an adverse event as "zero" unless it was explicitly stated as such in the article (15). We were unable to distinguish between sex and gender based on the included studies, so hereafter refer to sex/gender $(16,17)$.

For all studies, we examined the reported sex/gender distribution of the research participants. The number of participants in a study was derived from the number of participants reported at the study end point (specifically those on whom the study was conducted); if this was not available the number at study baseline was used. If data were presented by vaccine dose in the same participant group, then the number of participants in the second dose was extracted. Participant number from national surveillance data was taken as the number of vaccine recipients within the reporting period of the study (i.e., CDC reports: 14-23 December 2020 for Pfizer, and 21 December 2020 to 10 January 2021 for Moderna).

For all studies, we examined whether efficacy or effectiveness (hereafter efficacy) and safety data were presented by sex/gender. Studies were marked "Yes" for sex/gender-disaggregated data if they presented disaggregated data for all their reported main outcomes, either in the main results or Supplementary Information. For studies other than case series that disaggregated their safety findings by sex/gender, we either extracted relative risks of adverse events in women and men, or calculated them where possible from presented sex/gender-disaggregated participant and outcome data, in order to summarise the evidence for significant sex/gender differences.

No meta-analyses were performed owing to the relatively small number of studies available, and the heterogeneity in efficacy and safety outcomes that were reported.

\section{RESULTS}

A total of 323 relevant studies were identified, and 41 were eligible for inclusion in this review (Appendix Figure 1). Included studies presented data on the following vaccines: 
Oxford/AstraZeneca ( $n=11)$, Pfizer/BioNTech $(n=28)$, Moderna $(n=12)$, Johnson\&Johnson $(n=2)$, with some studies reporting data for more than one vaccine type.

Table 1 presents a summary of the content, participants, and presentation of outcomes in each of the included studies. Two adjusted for sex in their vaccine effectiveness model $(30,51)$, one study included sex-matched controls (43) and several articles were published as research letters and correspondence, rather than full research articles.

Across all seven RCTs, there was a 50/50 distribution in the sex/gender of participants (Table 1): one study included $44 \%$ women, four studies included $45-55 \%$ women, and two studies $\sim 60 \%$ women. A total of $3 / 11$ cohort studies included $45-55 \%$ women, with the other seven including more women (65-79\%), and one not reporting. In the case of cross-sectional studies, one study included $46 \%$ women and the remainder included $64-88 \%$ women. For routine surveillance reports, 5/8 (63\%) did not provide any sex/gender-disaggregated percentages of participants, and the remaining three included $61-62 \%$ women. Of the seven case studies, one did not provide disaggregated participant data, one included $100 \%$ women, and the rest included $40-$ $89 \%$ women.

Of the 10 studies investigating vaccine efficacy, three (two RCTs and one cohort) included sex/gender-disaggregated results (Table 1). None of these studies reported a significant difference in their primary efficacy outcome between women and men.

A range of local and systemic adverse reactions following immunisation were reported, as indicated in Table 1. Of the 35 experimental and observational studies containing safety data, $12(34 \%)$ disaggregated all their outcomes by sex/gender, none of which were RCTs and five of which were case series. An additional four studies reported sex/gender-disaggregated data for some but not all of their outcomes, and five additional studies presented summary statements by sex/gender, such as overall percentages of women and men experiencing at least one adverse event, or percentage requiring emergency department presentation. Sex/gender-specific risks of different adverse events were available from seven studies (Figure 1), which often indicated a higher risk reported for women. This included typical local and systemic reactions such as redness [RR $=1.97$ (95\% CI: 1.49-2.61)], swelling [RR $=2.24$ (95\% CI: $1.75-$ $2.88)]$, and fever [RR $=1.41$ (95\% CI: $1.31-1.51)]$ as well as nonanaphylaxis allergic reaction $[\mathrm{RR}=5.16$ (95\% CI: $2.49-10.70)$ for Pfizer/BioNTech vaccine and RR = 5.74 (95\% CI: 2.05-16.06) for Moderna vaccine]. Risk of one reaction, arterial event, appeared lower in women $[\mathrm{RR}=0.37$ (95\% CI: 0.24-0.57)].

No study justified lack of sex/gender-disaggregated data. However, two studies did acknowledge the dominance of women in their samples of healthcare workers $(23,30,40,43,51)$.

\section{DISCUSSION}

\section{Overall Summary of Findings}

In this mini review of studies reporting efficacy and/or safety outcomes of vaccines included under COVAX, we found that women and men were equally represented in RCTs, whilst women (and healthcare workers) comprised the majority of participants in observational studies. Despite global calls for the routine disaggregation of COVID-19 data by sex/gender (59-62), only two RCTs reported efficacy data by sex/gender, and none stratified safety data by sex/gender. Among the 34 included observational studies, 13 (38\%) presented all sex/gender-disaggregated data $(n=1$ (3\%) for efficacy and $n$ $=12(35 \%)$ for safety). There was no evidence of sex/gender differences in vaccine efficacy, yet a higher risk of adverse events were reported among women compared to men. However, this evidence was limited in terms of both number and size of studies, which may not have been specifically designed to detect a difference between groups.

Our review findings demonstrate a disappointing, and potentially detrimental, lack of sex/gender-specific evidence across study types of the COVID-19 vaccine experimental research studies as well as observational reporting.

\section{Representation by Sex/Gender in Research}

We found equal representation of women and men in COVID19 vaccine RCTs. This is despite another COVID-19 review finding that less than half of registered vaccine trials explicitly mentioned sex/gender in their recruitment strategy as part of their ClinicalTrials.gov registration (63). Therefore, our finding may be due to specific efforts by the research team to ensure equal recruitment, or because some of the usual barriers to women's participation in research, such as belief in the relevance of the health problem, concerns about risk, and trial logistical burden, may not have been as pervasive $(7,64)$. Women made up the majority of participants in non-RCT studies in our review. This is likely due, in part, to risk-based prioritisation of vaccine rollouts, which meant that healthcare and hospital workers, primarily women, were amongst the first to be vaccinated. For case series, it might reflect gender differences in reporting.

\section{Reporting of Sex/Gender-Disaggregated Data}

Our study identified a lack of sex/gender-disaggregated reporting or sub-group analyses in COVID-19 vaccine research. Despite roughly equal representation in RCTs, only a third of studies reported sex/gender-disaggregated efficacy data, and none reported safety data. This lack of focus on sex/gender aligns with findings of a recently published review of COVID-19 clinical trials of drug-based and biological/vaccine interventions, which found that only $18 \%$ of trials reported sex-disaggregated results or subgroup analyses (63). Another review published in early 2021 concluded that there was inadequate reporting of sex/ gender in COVID-19 clinical studies, that main outcomes were rarely reported or analysed by sex/gender, and this absence was seldom justified (65). We recognise the challenges in ensuring adequate sample sizes in clinical research to conduct subgroup analyses, particularly when considering rarer adverse events. However, the presentation of sex/gender-disaggregated data, either in main results or Supplementary Appendices as recommended in the SAGER guidelines (66), will be an asset for facilitating future meta-analyses as the pandemic progresses and the volume of COVID-19 vaccine research increases. 
TABLE 1 | Description of studies and their inclusion of sex/gender-disaggregated data.

\begin{tabular}{|c|c|c|c|c|c|c|c|c|}
\hline \multirow[b]{2}{*}{$\begin{array}{l}\text { Study Author (year) } \\
\text { Journal }\end{array}$} & \multirow[b]{2}{*}{$\begin{array}{l}\text { Population } \\
\text { subgroup (if any) }\end{array}$} & \multirow[b]{2}{*}{$\begin{array}{l}\text { Total } \mathrm{n} \text { per } \\
\text { study }\end{array}$} & \multirow[b]{2}{*}{$\begin{array}{l}\% . \text { of women } \\
\text { participants }\end{array}$} & \multicolumn{2}{|c|}{ Efficacy/Effectiveness data } & \multicolumn{3}{|c|}{ Safety data } \\
\hline & & & & $\begin{array}{c}\text { Contains } \\
\text { efficacy/ } \\
\text { effectiveness } \\
\text { data }\end{array}$ & $\begin{array}{l}\text { Sex/gender- } \\
\text { disaggregated } \\
\text { outcomes }\end{array}$ & $\begin{array}{l}\text { Contains } \\
\text { safety data }\end{array}$ & $\begin{array}{l}\text { Sex/gender- } \\
\text { disaggregated } \\
\text { outcomes }\end{array}$ & $\begin{array}{l}\text { List of adverse } \\
\text { reaction outcomes } \\
\text { investigated }\end{array}$ \\
\hline $\begin{array}{l}\text { Randomised controlled } \\
\text { trials }(n=7)\end{array}$ & & 88,255 & 50 & 6 & 2 & 5 & 0 & \\
\hline $\begin{array}{l}\text { Baden et al. (18) } \\
\text { N Engl J Med }\end{array}$ & - & 30,351 & 47 & Yes & Yes & Yes & No & $\begin{array}{l}\text { Local grade } 1,2 \text {, or } 3 \text { adverse reactions: } \\
\text { Any, pain, erythema, swelling, } \\
\text { axillary swelling/tenderness. } \\
\text { Systemic grade } 1,2 \text {, or } 3 \text { adverse } \\
\text { reactions: } \\
\text { Any, fever, headache, fatigue, myalgia, } \\
\text { arthralgia, nausea/vomiting, chills. }\end{array}$ \\
\hline $\begin{array}{l}\text { Emary et al. (19) } \\
\text { Lancet }\end{array}$ & - & 8,534 & 59 & Yes & No & No & NA & NA \\
\hline $\begin{array}{l}\text { Frenck et al. (20) } \\
\text { N Engl J Med }\end{array}$ & - & 2,260 & 49 & Yes & No & Yes & No & $\begin{array}{l}\text { Local mild, moderate, severe, and grade } 4 \\
\text { adverse reactions: } \\
\text { Pain at injection site, redness, swelling. } \\
\text { Systemic mild, moderate, severe and grade } \\
4 \text { adverse reactions: } \\
\text { Fever, fatigue, headache, chills, muscle } \\
\text { pain, vomiting, diarrhoea, joint pain. }\end{array}$ \\
\hline $\begin{array}{l}\text { Madhi et al. (21) } \\
\text { N Engl J Med }\end{array}$ & - & 2,021 & 43 & Yes & No & Yes & No & $\begin{array}{l}\text { General disorders, administration site } \\
\text { conditions, infections, nervous system, } \\
\text { respiratory, gastrointestinal, } \\
\text { musculoskeletal, skin, reproductive } \\
\text { system, eye, vascular, metabolism, ear, } \\
\text { immune system, renal, blood, psychiatric } \\
\text { disorders, and severe adverse events. }\end{array}$ \\
\hline $\begin{array}{l}\text { Polack et al. (22) } \\
\text { N Engl J Med }\end{array}$ & - & 37,706 & 49 & Yes & Yes & Yes & No & $\begin{array}{l}\text { Local mild, moderate, severe and grade } 4 \\
\text { adverse reactions: } \\
\text { Pain at injection site, redness, swelling. } \\
\text { Systemic mild, moderate, severe and grade } \\
4 \text { adverse reactions: } \\
\text { Fever, fatigue, headache, chills, vomiting, } \\
\text { diarrhoea, muscle pain, joint pain. }\end{array}$ \\
\hline $\begin{array}{l}\text { Ramasamy et al. (23) } \\
\text { Lancet }\end{array}$ & - & 552 & 51 & No & NA & Yes & No & $\begin{array}{l}\text { Local mild, moderate and severe adverse } \\
\text { reactions: } \\
\text { Induration, itch, pain, redness, swelling, } \\
\text { tenderness, warmth. } \\
\text { Systemic mild, moderate and severe } \\
\text { adverse reactions: } \\
\text { Chills, fatigue, fever, headache, joint pain, } \\
\text { malaise, muscle ache, nausea. }\end{array}$ \\
\hline $\begin{array}{l}\text { Voysey et al. (24) } \\
\text { Lancet }\end{array}$ & - & 6,831 & 62 & Yes & No & No & NA & NA \\
\hline
\end{tabular}

Population

of women

fety data

ex/gende

ectiveness

outcomes

outcomes

Randomised controlled

Lancet 


\begin{tabular}{|c|c|c|c|c|c|c|c|c|}
\hline \multirow[b]{2}{*}{$\begin{array}{l}\text { Study Author (year) } \\
\text { Journal }\end{array}$} & \multirow[b]{2}{*}{$\begin{array}{l}\text { Population } \\
\text { subgroup (if any) }\end{array}$} & \multirow[b]{2}{*}{$\begin{array}{l}\text { Total n per } \\
\text { study }\end{array}$} & \multirow[b]{2}{*}{$\begin{array}{l}\% . \text { of women } \\
\text { participants }\end{array}$} & \multicolumn{2}{|c|}{ Efficacy/Effectiveness data } & \multicolumn{3}{|c|}{ Safety data } \\
\hline & & & & $\begin{array}{c}\text { Contains } \\
\text { efficacy/ } \\
\text { effectiveness } \\
\text { data }\end{array}$ & $\begin{array}{l}\text { Sex/gender- } \\
\text { disaggregated } \\
\text { outcomes }\end{array}$ & $\begin{array}{l}\text { Contains } \\
\text { safety data }\end{array}$ & $\begin{array}{l}\text { Sex/gender- } \\
\text { disaggregated } \\
\text { outcomes }\end{array}$ & $\begin{array}{l}\text { List of adverse } \\
\text { reaction outcomes } \\
\text { investigated }\end{array}$ \\
\hline Cohort study $(n=11)$ & & $1,555,243$ & 56 & 2 & 1 & 9 & 4 & \\
\hline $\begin{array}{l}\text { Achiron et al. (25) } \\
\text { Mult Scler }\end{array}$ & $\begin{array}{l}\text { People with } \\
\text { multiple sclerosis }\end{array}$ & 435 & 65 & No & NA & Yes & No & $\begin{array}{l}\text { Pain at injection site, fever/chills/flu-like } \\
\text { symptoms, fatigue, headache, muscle or } \\
\text { joint pain, new or worsening neurological } \\
\text { symptomatology, face tingling, acute MS } \\
\text { relapse. }\end{array}$ \\
\hline $\begin{array}{l}\text { Bae et al. (26) } \\
\text { J Korean Med Sci }\end{array}$ & $\begin{array}{l}\text { Healthcare } \\
\text { workers }\end{array}$ & 5,866 & 76 & No & NA & Yes & Yes & $\begin{array}{l}\text { Local pain, redness, swelling, fever, } \\
\text { fatigue, headache, chills, vomiting, } \\
\text { diarrhoea, muscle ache, joint pain. }\end{array}$ \\
\hline $\begin{array}{l}\text { Bernstine et al. (27) } \\
\text { Clin Nucl Med }\end{array}$ & $\begin{array}{l}\text { People with } \\
\text { cancer }\end{array}$ & 256 & 54 & No & NA & Yes & Yes & Hypermetabolic axillary lymph nodes \\
\hline $\begin{array}{l}\text { Blumenthal et al. (28) } \\
\text { JAMA }\end{array}$ & Hospital workers & 64,900 & NR & No & NA & Yes & Yes & Anaphylaxis, acute allergic reactions \\
\hline $\begin{array}{l}\text { Dagan et al. (29) } \\
\text { N Engl J Med }\end{array}$ & $\begin{array}{l}\text { Health service } \\
\text { employees }\end{array}$ & $1,193,236$ & 50 & Yes & Yes & No & NA & NA \\
\hline $\begin{array}{l}\text { Fabiani et al. (30) } \\
\text { Euro Surveill }\end{array}$ & $\begin{array}{l}\text { Healthcare } \\
\text { workers }\end{array}$ & 6,423 & 78 & Yes & No & No & NA & NA \\
\hline $\begin{array}{l}\text { Jeon et al. (31) } \\
\text { J Korean Med Sci }\end{array}$ & $\begin{array}{l}\text { Healthcare } \\
\text { workers }\end{array}$ & 994 & 77 & No & NA & Yes & Some $^{+}$ & $\begin{array}{l}\text { Fatigue, headache, malaise, arthralgia, } \\
\text { chills, fever, nausea/vomiting, diarrhoea, } \\
\text { local tenderness, redness, swelling, } \\
\text { resting pain. }\end{array}$ \\
\hline $\begin{array}{l}\text { Kim et al. (32) } \\
\text { J Korean Med Sci }\end{array}$ & $\begin{array}{l}\text { Healthcare } \\
\text { workers }\end{array}$ & 1,511 & 72 & No & NA & Yes & Some $^{\star+}$ & $\begin{array}{l}\text { Pain at injection site, redness/swelling at } \\
\text { injection site, lymphadenopathy, fever, } \\
\text { chills, fatigue, nausea, vomiting, } \\
\text { headache, myalgia, arthralgia, urticaria. }\end{array}$ \\
\hline $\begin{array}{l}\text { Krammer et al. (33) } \\
\text { N Engl J Med }\end{array}$ & - & 230 & 68 & No & NA & Yes & No & $\begin{array}{l}\text { Pain at injection site, swelling at injection } \\
\text { site, erythema, fatigue, headache, chills, } \\
\text { muscle pain, fever, joint pain. }\end{array}$ \\
\hline $\begin{array}{l}\text { Pimpinelli et al. (34) } \\
\text { J Hematol Oncol }\end{array}$ & $\begin{array}{l}\text { People with } \\
\text { hematologic } \\
\text { malignancies }\end{array}$ & 128 & 48 & No & NA & Yes & No & $\begin{array}{l}\text { Pain, tenderness, fever, headache, } \\
\text { malaise, myalgia, chills. }\end{array}$ \\
\hline $\begin{array}{l}\text { Pottegard et al. (35) } \\
\text { BMJ }\end{array}$ & - & 281,264 & 79 & No & NA & Yes & Yes & $\begin{array}{l}\text { Arterial events, venous } \\
\text { thromboembolism,/coagulation disorders, } \\
\text { bleeding events. }\end{array}$ \\
\hline $\begin{array}{l}\text { Cross-sectional survey ( } n \\
=8)\end{array}$ & & 7,243 & 77 & & & 8 & 0 & \\
\hline $\begin{array}{l}\text { Boyarsky et al. (36) } \\
\text { Transplantation }\end{array}$ & $\begin{array}{l}\text { Solid organ } \\
\text { transplant } \\
\text { recipients }\end{array}$ & 187 & 69 & No & NA & Yes & No & $\begin{array}{l}\text { Pain, redness, swelling, fever, chills, } \\
\text { fatigue, headache, myalgia. }\end{array}$ \\
\hline
\end{tabular}




\begin{tabular}{|c|c|c|c|c|c|c|c|c|}
\hline \multirow[b]{2}{*}{$\begin{array}{l}\text { Study Author (year) } \\
\text { Journal }\end{array}$} & \multirow[b]{2}{*}{$\begin{array}{l}\text { Population } \\
\text { subgroup (if any) }\end{array}$} & \multirow[b]{2}{*}{$\begin{array}{l}\text { Total } n \text { per } \\
\text { study }\end{array}$} & \multirow[b]{2}{*}{$\begin{array}{l}\% \text {. of women } \\
\text { participants }\end{array}$} & \multicolumn{2}{|c|}{ Efficacy/Effectiveness data } & \multicolumn{3}{|c|}{ Safety data } \\
\hline & & & & $\begin{array}{c}\text { Contains } \\
\text { efficacy/ } \\
\text { effectiveness } \\
\text { data }\end{array}$ & $\begin{array}{l}\text { Sex/gender- } \\
\text { disaggregated } \\
\text { outcomes }\end{array}$ & $\begin{array}{l}\text { Contains } \\
\text { safety data }\end{array}$ & $\begin{array}{l}\text { Sex/gender- } \\
\text { disaggregated } \\
\text { outcomes }\end{array}$ & $\begin{array}{l}\text { List of adverse } \\
\text { reaction outcomes } \\
\text { investigated }\end{array}$ \\
\hline $\begin{array}{l}\text { El-Shitany et al. (37) } \\
\text { Int J Gen Med }\end{array}$ & - & 455 & 64 & No & NA & Yes & Some $^{+}$ & $\begin{array}{l}\text { Arm pain, injection site pain, injection site } \\
\text { swelling and redness, fever, chills, fatigue, } \\
\text { headache, nausea and vomiting, } \\
\text { diarrhoea, muscle pain, joint pain. }\end{array}$ \\
\hline $\begin{array}{l}\text { Kadali et al. (38) } \\
\text { Int } J \text { Infect Dis }\end{array}$ & $\begin{array}{l}\text { Healthcare } \\
\text { workers }\end{array}$ & 803 & 87 & No & NA & Yes & No & $\begin{array}{l}\text { Generalised, weakness/fatigue, headache, } \\
\text { chills, localised swelling at injection site, } \\
\text { muscle pain/myalgia, arthritis/joint pain, } \\
\text { diarrhoea, vomiting, fever, nausea, sore } \\
\text { arm/pain, sweating. }\end{array}$ \\
\hline $\begin{array}{l}\text { Nittner-Marzalska et al. (39) } \\
\text { Vaccines }\end{array}$ & $\begin{array}{l}\text { Medical } \\
\text { professionals and } \\
\text { medical students }\end{array}$ & 1,707 & 79 & No & NA & Yes & No & $\begin{array}{l}\text { Fever, arthralgia, myalgia, headache, } \\
\text { palpitations, vomiting, local swelling, local } \\
\text { redness, local pain, allergic reactions. }\end{array}$ \\
\hline $\begin{array}{l}\text { Riad et al. (40) } \\
\text { J Clin Med }\end{array}$ & $\begin{array}{l}\text { Healthcare } \\
\text { workers }\end{array}$ & 877 & 88 & No & NA & Yes & No & $\begin{array}{l}\text { General side effects: injection site pain, } \\
\text { fatigue, headache, muscle pain, chills, joint } \\
\text { pain, injection site swelling, injection site } \\
\text { redness feeling unwell, lymphadenopathy, } \\
\text { nausea. } \\
\text { Oral side effects }\end{array}$ \\
\hline $\begin{array}{l}\text { Song et al. (41) } \\
\text { J Korean Med Sci }\end{array}$ & $\begin{array}{l}\text { Healthcare } \\
\text { workers }\end{array}$ & 2,478 & 76 & No & NA & Yes & No & $\begin{array}{l}\text { Injection site pain, injection site erythema, } \\
\text { fever, headache, myalgia, arthralgia, } \\
\text { fatigue, nausea/vomiting, rash, limitation of } \\
\text { arm movement, facial paraesthesia, chill. } \\
\text { Anaphylactoid reaction. }\end{array}$ \\
\hline $\begin{array}{l}\text { Sørvoll et al. (42) } \\
\text { J Thromb Haemost }\end{array}$ & $\begin{array}{l}\text { Healthcare } \\
\text { workers }\end{array}$ & 602 & 71 & No & NA & Yes & No & $\begin{array}{l}\text { Thrombocytopenia, anti-PF4/PVS reaction } \\
\text { antibodies. } \\
\text { Fever, headache, vomiting, fatigue, } \\
\text { cutaneous bleeding, malaise, } \\
\text { muscle/joint ache. }\end{array}$ \\
\hline $\begin{array}{l}\text { Waissengren et al. (43) } \\
\text { Lancet Oncol }\end{array}$ & $\begin{array}{l}\text { People with } \\
\text { cancer }\end{array}$ & 134 & 46 & No & NA & Yes & No & $\begin{array}{l}\text { Pain at injection sites, fatigue, headache, } \\
\text { muscle pain, chills, fever, gastrointestinal } \\
\text { complications, flu-like symptoms, local } \\
\text { rash, local swelling. }\end{array}$ \\
\hline $\begin{array}{l}\text { Routine surveillance }(n= \\
\text { 8) }\end{array}$ & & $41,104,426$ & 61 & 2 & 0 & 6 & 3 & \\
\hline $\begin{array}{l}\text { CDC COVID-19 Response } \\
\text { Team et al. (44) } \\
\text { Morb Mortal Wkly Rep }\end{array}$ & VAERS & $1,893,360$ & 62 & No & NA & Yes & Yes & $\begin{array}{l}\text { Anaphylaxis, non-anaphylaxis allergic } \\
\text { reactions }\end{array}$ \\
\hline $\begin{array}{l}\text { CDC COVID-19 Response } \\
\text { Team et al. (45) } \\
\text { Morb Mortal Wkly Rep }\end{array}$ & VAERS & $4,041,396$ & 61 & No & NA & Yes & Yes & $\begin{array}{l}\text { Anaphylaxis, non-anaphylaxis allergic } \\
\text { reactions }\end{array}$ \\
\hline
\end{tabular}




\begin{tabular}{|c|c|c|c|c|c|c|c|c|}
\hline \multirow[b]{2}{*}{$\begin{array}{l}\text { Study Author (year) } \\
\text { Journal }\end{array}$} & \multirow[b]{2}{*}{$\begin{array}{l}\text { Population } \\
\text { subgroup (if any) }\end{array}$} & \multirow[b]{2}{*}{$\begin{array}{l}\text { Total } \mathrm{n} \text { per } \\
\quad \text { study }\end{array}$} & \multirow[b]{2}{*}{$\begin{array}{l}\text { \%. of women } \\
\text { participants }\end{array}$} & \multicolumn{2}{|c|}{ Efficacy/Effectiveness data } & \multicolumn{3}{|c|}{ Safety data } \\
\hline & & & & $\begin{array}{l}\text { Contains } \\
\text { efficacy/ } \\
\text { effectiveness } \\
\text { data }\end{array}$ & $\begin{array}{l}\text { Sex/gender- } \\
\text { disaggregated } \\
\text { outcomes }\end{array}$ & $\begin{array}{l}\text { Contains } \\
\text { safety data }\end{array}$ & $\begin{array}{l}\text { Sex/gender- } \\
\text { disaggregated } \\
\text { outcomes }\end{array}$ & $\begin{array}{l}\text { List of adverse } \\
\text { reaction outcomes } \\
\text { investigated }\end{array}$ \\
\hline $\begin{array}{l}\text { Gee et al. (46) } \\
\text { Morb Mortal Wkly Rep }\end{array}$ & VAERS and V-safe & $1,629,065$ & NR & No & NA & Yes & Some $^{+}$ & $\begin{array}{l}\text { Pain at injection site, fatigue, headache, } \\
\text { myalgia, chills, fever, swelling at injection } \\
\text { site, joint pain, nausea. }\end{array}$ \\
\hline $\begin{array}{l}\text { Hause et al. (47) Morb } \\
\text { Mortal Wkly Rep }\end{array}$ & VAERS & $7,988,624$ & NR & No & NA & Yes & Some $^{+}$ & $\begin{array}{l}\text { Anxiety related adverse events: chest pain, } \\
\text { light-headedness or dizziness, } \\
\text { nausea/vomiting, pallor or diaphoresis, } \\
\text { syncope, tachycardia, seizure-like activity, } \\
\text { hypotension. }\end{array}$ \\
\hline $\begin{array}{l}\text { Shay et al. (48) } \\
\text { Morb Mortal Wkly Rep }\end{array}$ & VAERS and V-safe & $7,980,000$ & NR & No & NA & Yes & Some ${ }^{*}$ & $\begin{array}{l}\text { Non-serious, serious events, injection site } \\
\text { reactions, systemic reactions, health } \\
\text { impacts, fatigue, injection site pain, } \\
\text { headache, myalgia, fever, joint pain, } \\
\text { nausea, diarrhoea. }\end{array}$ \\
\hline $\begin{array}{l}\text { Shimabukuro et al. (49) } \\
\text { JAMA }\end{array}$ & VAERS & $17,524,676$ & NR & No & NA & Yes & Yes & Anaphylaxis \\
\hline $\begin{array}{l}\text { Skowronski et al. (50) } \\
\text { N Engl J Med }\end{array}$ & $\begin{array}{l}\text { Documents } \\
\text { submitted to Food } \\
\text { and Drug } \\
\text { Administration }\end{array}$ & 43,355 & NR & Yes & No & No & NA & NA \\
\hline $\begin{array}{l}\text { Thompson et al. (51) } \\
\text { Morb Mortal Wkly Rep }\end{array}$ & $\begin{array}{l}\text { Healthcare } \\
\text { workers in } \\
\text { HEROES- } \\
\text { RECOVER }\end{array}$ & 3,950 & 62 & Yes & No & No & NA & NA \\
\hline Case Series $(n=7)$ & & 154 & 71 & & & 7 & 5 & \\
\hline $\begin{array}{l}\text { Farinazzo et al. (52) } \\
\text { J Eur Acad Dermatol } \\
\text { Venereol }\end{array}$ & - & 46 & 89 & No & NA & Yes & Yes & $\begin{array}{l}\text { Cutaneous adverse reaction, any adverse } \\
\text { event }\end{array}$ \\
\hline $\begin{array}{l}\text { Fernandez-Prada et al. (53) } \\
\text { Euro Surveill }\end{array}$ & $\begin{array}{l}\text { Healthcare } \\
\text { workers }\end{array}$ & 20 & 100 & No & NA & Yes & Yes & Supraclavicular lymphadenopathy \\
\hline $\begin{array}{l}\text { Johnston et al. (54) } \\
\text { JAMA Dermatol }\end{array}$ & - & 16 & 81 & No & NA & Yes & Yes & $\begin{array}{l}\text { Localised cutaneous reaction (injection } \\
\text { site reactions) }\end{array}$ \\
\hline $\begin{array}{l}\text { Lee et al. (55) } \\
\text { Am J Hematol }\end{array}$ & - & 20 & 40 & No & NA & Yes & Some ${ }^{\star}$ & Thrombocytopenia, bruising, bleeding \\
\hline $\begin{array}{l}\text { Meylan et al. (56) } \\
\text { Hypertension }\end{array}$ & - & 9 & 78 & No & NA & Yes & Yes & Stage 3 hypertension \\
\hline $\begin{array}{l}\text { Roman et al. (57) } \\
\text { Front Immunol }\end{array}$ & - & 43 & 47 & No & NA & Yes & Some ${ }^{*}$ & $\begin{array}{l}\text { Quadriplegia, paraplegia, acute } \\
\text { disseminated encephalomyelitis, spinal } \\
\text { cord lesions }\end{array}$ \\
\hline $\begin{array}{l}\text { Shemer et al. (58) } \\
\text { Isr Med Assoc J }\end{array}$ & - & 9 & NR & No & NA & Yes & Yes & Acute-onset facial nerve palsy \\
\hline
\end{tabular}

NR, Not reported; NA, not applicable.

"Study sex/gender-disaggregated presentation of some but not all safety outcomes.

+ Study presented sex/gender-disaggregated summary results. 


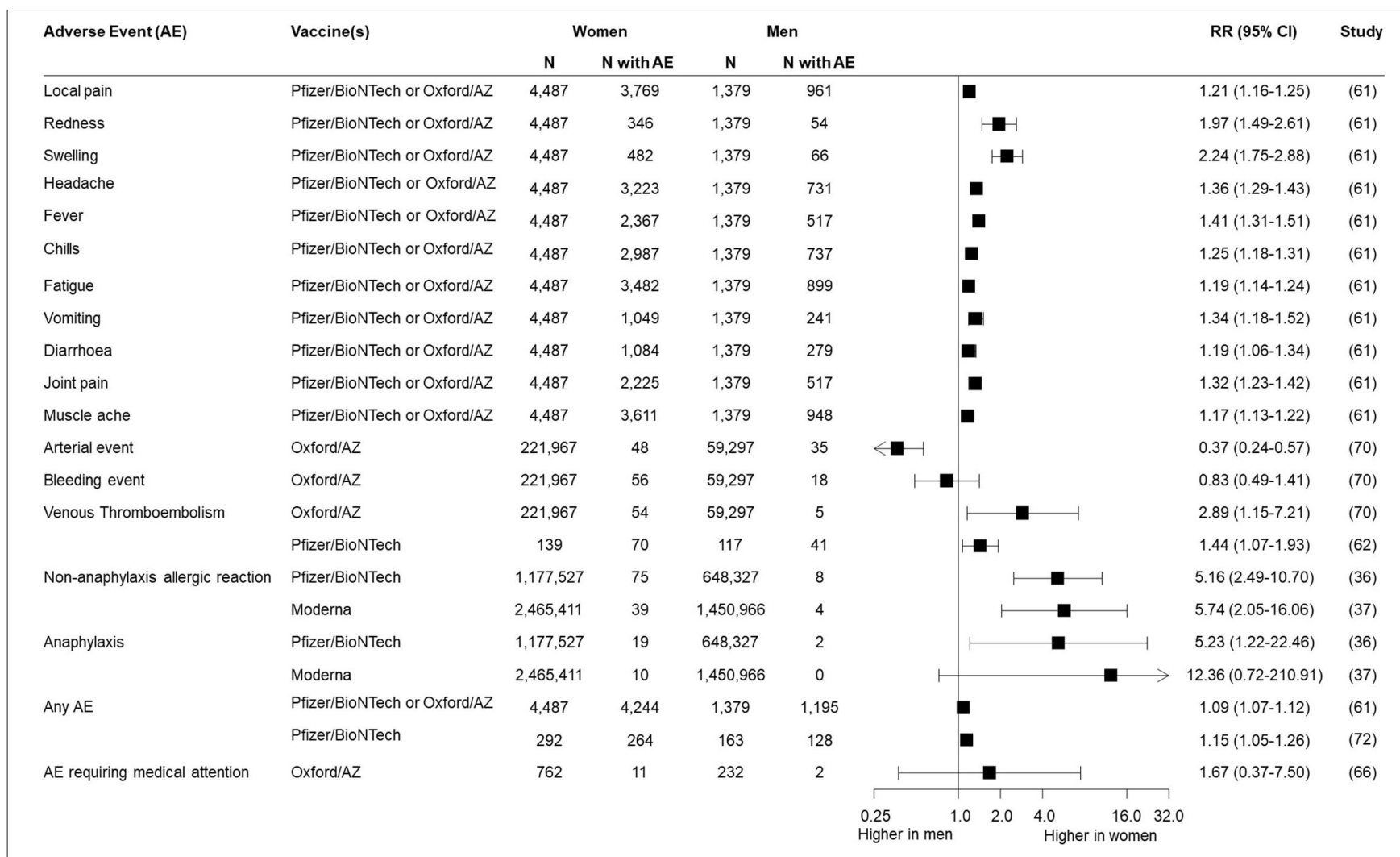

FIGURE 1 | Risk of adverse events following COVID-19 immunisation reported in women and men.

A novel element of our review is the inclusion of multiple study designs, not only RCTs. The complete absence of sex/gender-disaggregated safety data in COVID-19 vaccine trials means that post-marketing surveillance of sex/gender-specific adverse events is particularly important. Yet there was still an absence of reporting, with sex/gender-disaggregated adverse event data available in only four cohort and three national surveillance studies. While age and sex/gender data are typically collected through routine national surveillance systems (67), and shared with decision makers, the lack of data in the public domain has consequences for immunisation program delivery and uptake - further discussed below.

\section{Evidence of Sex/Gender Differences}

Of the seven studies where sex/gender-specific risk was reported, a higher risk of certain types of adverse events were observed among women. A large prospective observational study published subsequently to our searches found that local and systemic side effects were self-reported at lower frequencies than reported in RCTs, and minor events such as headache and fatigue were more common in women (68). These findings align with that of other vaccines (69), and are likely primarily due to women being more likely to report their symptoms than men $(70,71)$. Looking at anaphylactic and non-anaphylactic allergic reactions, although rare, $90 \%$ were reported in women $(44,45,49)$. This is likely influenced by greater percentage of women being vaccinated (45). These findings raise important questions around the gendered dimensions of immunisation, and demonstrate the value of routine collection and analysis of sex/gender-disaggregated data for further investigation of trends and mitigation strategies.

\section{Consequences of a Lack of Sex/Gender Specific Evidence}

Lack of sex/gender data in immunisation, including efficacy, safety and coverage data, has been a longstanding issue, not only one of the COVID-19 pandemic. Yet sex/gender has a critical influence on immunisation outcomes, at individual, household, community, health system, and policy levels (72). The lack of incorporation of sex/gender in COVID-19 vaccine research, as demonstrated across the spectrum of research designs in this review, results in an evidence base that does not lend itself to effective public communication around the utility and safety of vaccines. One current example is vaccine hesitancy and the slow uptake in some regions $(73,74)$. While a complex issue with many inter-related factors, concerns around safety (including pain) and misconceptions about effectiveness and side effects are known to be critical influences of vaccine hesitancy and delay $(6,75)$. Previous research has fairly consistently found that men report a higher intention to vaccinate than women (7678), though intention does not always reflect action or access. 
Therefore, an increase in sex/gender-specific information in the public domain would appear to be a prudent approach so as to help address misconceptions and mitigate vaccine hesitancy. This tailored advice is only possible if research pays more attention to sex/gender, and the particular concerns of women, men, and non-binary people.

A gendered lens should also be applied when designing research, including determining what data to collect. This includes consideration of how research design and conduct may be explicitly or implicitly sex/gender-biased, for example through exclusion of those who are pregnant or breastfeeding (79), or how research may potentially exacerbate existing sex/genderrelated disparities or knowledge gaps. As an example, only one of the included studies examined adverse events related to the reproductive system, and the authors did not disaggregate these findings by sex/gender, or by age (21). Surveillance studies have also not reported data on menstrual irregularities or fertility. This may limit the ability of scientists and doctors to effectively respond to anecdotal reports and concerns within the community about such side effects post-immunisation, which have been amplified online and by the media (80-83). Greater attention to sex/gender in the design of COVID-19 vaccine research may help to address this data gap, allowing for improved public communication about adverse events with patientsparticularly women.

\section{RECOMMENDATIONS FOR FUTURE IMMUNISATION RESEARCH AND SURVEILLANCE}

There are some limitations to this mini-review. In particular, the potential for missing studies, as only one database was searched and COVID-19 publications are rapidly increasing in number over time. However, this snapshot demonstrates an important evidence gap and discusses how adopting a sex/gender lens to data collection, reporting and analysis can have benefits for vaccination program outcomes. This mini-review focused only on sex/gender, without incorporating other intersectional factors, such as age and ethnicity, that may have an important impact on COVID-19 immunisation and outcomes and should be incorporated into research. Further, a quality assessment of articles was not conducted, which could be relevant for studies reporting sex/gender differences in outcomes, particularly regarding sample size and gender distribution. However, this mini review highlights substantial gaps in sex/gender-specific COVID-19 vaccine research.

Sex/gender, and other intersecting factors, impact how people are experiencing the COVID-19 pandemic (84). Therefore, research, policy and recommendations for COVID-19 vaccination must consider sex/gender in order to achieve optimally effective and equitable outcomes. Based on our findings, we present the following recommendations for future research:

- All research studies should, within reason, include a sex/gender lens in their research design and recruitment, sex/gender-disaggregate their main outcomes and, where feasible, analyse potential sex/gender-based differences, or indeed similarities. This aligns with advice provided to the WHO on critical considerations for equitable COVID-19 vaccine research, development, and delivery (6).

- Data from research studies that collect the sex/gender of participants, but are not statistically powered to analyse results by sex/gender, should nevertheless make sex/gender results publicly available for pooling in evidence syntheses, even if only through Supplementary Data published online $(66,85)$.

- Medical journals and editors, as well as public health bodies, should redouble their efforts in enforcing recognition of sex/gender in reporting of COVID-19 research $(65,86)$ including enforcement of policies or endorsed guidelines and instructions and advice for peer reviewers

- Public health data systems, processes, and platforms should be established or adapted to collect, publicly report, and reflect on sex/gender-disaggregated outcomes from nationwide/mass immunisation programs (87).

\section{CONCLUSION}

Studies developing new vaccines or investigating their impact in populations should be designed and implemented in a sex/gender-sensitive way. Failure to recognise important sex/gender implications on efficacy, safety, and implementation will be detrimental to the global vaccine rollout, and ultimately control of the COVID-19 pandemic. Review of clinical trial data highlights missed opportunities to apply a sex/gendersensitive lens in the development of COVID-19 vaccines. Public health data gathered through routine surveillance should be sex/gender-disaggregated and made publicly available to increase reliability of data, drive public confidence in immunisation programs, decrease vaccine hesitancy, and increase coverage.

\section{AUTHOR CONTRIBUTIONS}

AV conceived the study and wrote the initial draft. SS designed and conducted the searches in consultation with $\mathrm{AV}$. $\mathrm{AV}, \mathrm{SS}, \mathrm{KH}, \mathrm{LH}$, and $\mathrm{CH}$ conducted the data screening, extraction, and analysis. SS, KH, LH, $\mathrm{CH}$, MW, KW, and MS critically reviewed the study plan and manuscript and rewrote sections. All authors contributed to manuscript revisions, read, and approved the submitted version.

\section{FUNDING}

MS was funded by a Westpac Research Fellowship.

\section{SUPPLEMENTARY MATERIAL}

The Supplementary Material for this article can be found online at: https://www.frontiersin.org/articles/10.3389/fgwh. 2021.761511/full\#supplementary-material 


\section{REFERENCES}

1. Klein SL, Dhakal S, Ursin RL, Deshpande S, Sandberg K, MauvaisJarvis F. Biological sex impacts COVID-19 outcomes. PLoS Pathog. (2020) 16:e1008570. doi: 10.1371/journal.ppat.1008570

2. Global Health 5050. The Sex, Gender and COVID-19 Project. (2021). Available online at: https://globalhealth5050.org/the-sex-gender-and-covid19-project/ (accessed June 25, 2021)

3. Abate BB, Kassie AM, Kassaw MW, Aragie TG, Masresha SA. Sex difference in coronavirus disease (COVID-19): a systematic review and meta-analysis. BMJ Open. (2020) 10:e040129. doi: 10.1136/bmjopen-2020-040129

4. Peckham H, de Gruijter NM, Raine C, Radziszewska A, Ciurtin C, Wedderburn LR, et al. Male sex identified by global COVID-19 metaanalysis as a risk factor for death and ITU admission. Nat Commun. (2020) 11:6317. doi: 10.1038/s41467-020-19741-6

5. Walter LA, McGregor AJ. Sex-and gender-specific observations and implications for COVID-19. West J Emerg Med. (2020) 21:507. doi: 10.5811/westjem.2020.4.47536

6. Heidari S, Goodman T. Critical Sex and Gender Considerations for Equitable Research, Development and Delivery of COVID-19 Vaccines. World Health Oganization (2021). Available online at: https://cdn.who.int/media/docs/ default-source/immunization/sage/covid/gender-covid-19-vaccines-sagebackground-paper.pdf?sfvrsn=899e8fca_15\&download=true (accessed June 25, 2021).

7. Steinberg JR, Turner BE, Weeks BT, Magnani CJ, Wong BO, Rodriguez F, et al. Analysis of female enrollment and participant sex by burden of disease in US clinical trials between 2000 and 2020. JAMA Netw Open. (2021) 4:e2113749. doi: 10.1001/jamanetworkopen.2021.13749

8. Wolfe J, Safdar B, Madsen TE, Sethuraman KN, Becker B, Greenberg MR, et al. Sex-or gender-specific differences in the clinical presentation, outcome, and treatment of Sars-Cov2. Clin Ther. (2021) 43:557-71. doi: 10.1016/j.clinthera.2021.01.015

9. Fischinger S, Boudreau CM, Butler AL, Streeck H, Alter G. Sex differences in vaccine-induced humoral immunity. Semin Immunopathol. (2019) 41:23949. doi: 10.1007/s00281-018-0726-5

10. Klein SL, Marriott I, Fish EN. Sex-based differences in immune function and responses to vaccination. Trans R Soc Trop Med Hyg. (2015) 109:915. doi: $10.1093 /$ trstmh/tru167

11. Klein SL, Flanagan KL. Sex differences in immune responses. Nat Rev Immunol. (2016) 16:626-38. doi: 10.1038/nri.2016.90

12. Kim JH, Marks F, Clemens JD. Looking beyond COVID-19 vaccine phase 3 trials. Nat Med. (2021) 27:205-11. doi: 10.1038/s41591-02101230-y

13. World Health Organization. Status of COVID-19 Vaccines Within WHO EUL/PQ Evaluation Process. (2021). Available online at: https://extranet.who. int/pqweb/sites/default/files/documents/Status_COVID_VAX_04May2021. pdf (accessed May 2021)

14. World Health Organisation. COVAX. (2021). Available online at: https://www. who.int/initiatives/act-accelerator/covax (accessed June 2021).

15. Peryer G, Golder S, Junqueira D, Vohra S, Loke YK. Chapter 19: Adverse effects. In: Higgins JPT, Thomas J, Chandler J, Cumpston M, Li T, Page MJ, Welch VA, editors. Cochrane Handbook for Systematic Reviews of Interventions Version 6.2 (updated February 2021). Cochrane (2021). Available online at: www.training.cochrane.org/handbook

16. Van Anders SM. Beyond sexual orientation: integrating gender/sex and diverse sexualities via sexual configurations theory. Arch Sex Behav. (2015) 44:1177-213. doi: 10.1007/s10508-0150490-8

17. GenderSci Lab. Gender/sex: An Explanation. (2020). Available online at: https://www.genderscilab.org/blog/covid-intro (accessed July, 2021)

18. Baden LR, El Sahly HM, Essink B, Kotloff K, Frey S, Novak R, et al. Efficacy and safety of the mRNA-1273 SARS-CoV-2 vaccine. N Engl J Med. (2021) 384:403-16. doi: 10.1056/NEJMoa2035389

19. Emary KRW, Golubchik T, Aley PK, Ariani CV, Angus B, Bibi $\mathrm{S}$, et al. Efficacy of ChAdOxl nCoV-19 (AZD1222) vaccine against SARS-CoV-2 variant of concern 202012/01 (B.1.1.7): an exploratory analysis of a randomised controlled trial. Lancet. (2021) 397:1351-62. doi: 10.1016/S0140-6736(21)00628-0
20. Frenck RW Jr, Klein NP, Kitchin N, Gurtman A, Absalon J, Lockhart S, et al. Safety, immunogenicity, and efficacy of the BNT162b2 Covid-19 vaccine in adolescents. N Engl J Med. (2021) 385:239-50. doi: 10.1056/NEJMoa2107456

21. Madhi SA, Baillie V, Cutl, CL, Voysey M, Koen AL, et al. Efficacy of the ChAdOx1 nCoV-19 Covid-19 vaccine against the B.1.351 variant. $N$ Engl J Med. (2021) 384:1885-98. doi: 10.1056/NEJMoa2102214

22. Polack FP, Thomas SJ, Kitchin N, Absalon J, Gurtman A, Lockhart S, et al. Safety and efficacy of the BNT162b2 mRNA Covid-19 vaccine. N Engl J Med. (2020) 383:2603-15. doi: 10.1056/NEJMoa2034577

23. Ramasamy MN, Minassian AM, Ewer KJ, Flaxman AL, Folegatti PM, Owens DR, et al. Safety and immunogenicity of ChAdOx1 nCoV-19 vaccine administered in a prime-boost regimen in young and old adults (COV002): a single-blind, randomised, controlled, phase $2 / 3$ trial. Lancet. (2020) 396:1979-93. doi: 10.1016/S0140-6736(20)32466-1

24. Voysey M, Clemens SAC, Madhi SA, Weckx LY, Folegatti PM, Aley PK, et al. Safety and efficacy of the ChAdOx1 nCoV-19 vaccine (AZD1222) against SARS-CoV-2: an interim analysis of four randomised controlled trials in Brazil, South Africa, and the UK. Lancet. (2020) 397:99111. doi: $10.1016 / \mathrm{S} 0140-6736(20) 32661-1$

25. Achiron A, Dolev M, Menascu S, Zohar DN, Dreyer-Alster S, Miron $S$, et al. COVID-19 vaccination in patients with multiple sclerosis: what we have learnt by February 2021. Mult Scler. (2021) 27:86470. doi: 10.1177/13524585211003476

26. Bae S, Lee YW, Lim SY, Lee JH, Lim JS, Lee S, et al. Adverse reactions following the first dose of ChAdOx1 nCoV-19 vaccine and BNT162b2 vaccine for healthcare workers in South Korea. J Korean Med Sci. (2021) 36:e115. doi: 10.3346/jkms.2021.36.e115

27. Bernstine H, Priss M, Anati T, Turko O, Gorenberg M, Steinmetz AP, et al. Axillary lymph nodes hypermetabolism after BNT162b2 mRNA COVID-19 Vaccination in cancer patients undergoing 18F-FDG PET/CT: a cohort study. Clin Nucl Med. (2021) 46:396-401. doi: 10.1097/RLU.0000000000003648

28. Blumenthal KG, Robinson LB, Camargo CA Jr, Shenoy ES, Banerji A, et al. Acute allergic reactions to mRNA COVID-19 vaccines. JAMA. (2021) 325:1562-5. doi: 10.1001/jama.2021.3976

29. Dagan N, Barda N, Kepten E, Miron O, Perchik S, Katz MA, et al. BNT162b2 mRNA Covid-19 vaccine in a nationwide mass vaccination setting. $N$ Engl J Med. (2021) 384:1412-23. doi: 10.1056/NEJMoa2101765

30. Fabiani M, Ramigni M, Gobbetto V, Mateo-Urdiales A, Pezzotti P, Piovesan C. Effectiveness of the Comirnaty (BNT162b2, BioNTech/Pfizer) vaccine in preventing SARS-CoV-2 infection among healthcare workers, Treviso province, Veneto region, Italy, 27 December 2020 to 24 March 2021. Euro Surveill. (2021) 26:2100420. doi: 10.2807/1560-7917.ES.2021.26.17.2100420

31. Jeon M, Kim J, Oh CE, Lee JY. Adverse events following immunization associated with coronavirus disease 2019 vaccination reported in the mobile vaccine adverse events reporting system. J Korean Med Sci. (2021) 36:e114. doi: 10.3346/jkms.2021.36.e114

32. Kim SH, Wi YM, Yun SY, Ryu JS, Shin JM, Lee EH, et al. Adverse events in healthcare workers after the first dose of ChAdOx1 nCoV-19 or BNT162b2 mRNA COVID-19 vaccination: a single center experience. J Korean Med Sci. (2021) 36:e107. doi: 10.3346/jkms.2021.36.e107

33. Krammer F, Srivastava K, Alshammary H, Amoako AA, Awawda MH, Beach KF, et al. Antibody responses in seropositive persons after a single dose of SARS-CoV-2 mRNA vaccine. N Engl J Med. (2021) 384:13724. doi: $10.1056 / \mathrm{NEJMc} 2101667$

34. Pimpinelli F, Marchesi F, Piaggio G, Giannarelli D, Papa E, Falcucci $\mathrm{P}$, et al. Fifth-week immunogenicity and safety of anti-SARS-CoV-2 BNT162b2 vaccine in patients with multiple myeloma and myeloproliferative malignancies on active treatment: preliminary data from a single institution. $J$ Hematol Oncol. (2021) 14:81. doi: 10.1186/s13045-021-01090-6

35. Pottegård A, Lund LC, Karlstad $\varnothing$, Dahl J, Andersen M, Hallas J, et al. Arterial events, venous thromboembolism, thrombocytopenia, and bleeding after vaccination with Oxford-AstraZeneca ChAdOx1-S in Denmark and Norway: population based cohort study. BMJ. (2021) 373:n1114. doi: 10.1136/bmj.n1114

36. Boyarsky BJ, Ou MT, Greenberg RS, Teles AT, Werbel WA, Avery $\mathrm{RK}$, et al. Safety of the first dose of SARS-CoV-2 vaccination in solid organ transplant recipients. Transplantation. (2021) 105:e56-7. doi: 10.1097/TP.0000000000003654 
37. El-Shitany NA, Harakeh S, Badr-Eldin SM, Bagher AM, Eid B, Almukadi H, et al. Minor to moderate side effects of Pfizer-BioNTech COVID-19 vaccine among saudi residents: a retrospective cross-sectional study. Int J Gen Med. (2021) 14:1389-401. doi: 10.2147/IJGM.S310497

38. Kadali RAK, Janagama R, Peruru S, Malayala SV. Side effects of BNT162b2 mRNA COVID-19 vaccine: a randomized, cross-sectional study with detailed self-reported symptoms from healthcare workers. Int J Infect Dis. (2021) 106:376-81. doi: 10.1016/j.ijid.2021.04.047

39. Nittner-Marszalska M, Rosiek-Biegus M, Kopeć A, Pawłowicz R, Kosińska M, Łata A, et al. Pfizer-BioNTech COVID-19 vaccine tolerance in allergic versus non-allergic individuals. Vaccines. (2021) 9:553. doi: 10.3390/vaccines 9060553

40. Riad A, Pokorná A, Attia S, Klugarová J, Koščík M, Klugar M. Prevalence of COVID-19 vaccine side effects among healthcare workers in the Czech Republic. J Clin Med. (2021) 10:1428. doi: 10.3390/jcm10071428

41. Song JY, Cheong HJ, Kim SR, Lee SE, Kim SH, Noh JY, et al. Early safety monitoring of COVID-19 vaccines in healthcare workers. J Korean Med Sci. (2021) 36:e110. doi: 10.3346/jkms.2021.36.e110

42. Sørvoll IH, Horvei KD, Ernstsen SL, Laegreid IJ, Lund S, Grønli RH, et al. An observational study to identify the prevalence of thrombocytopenia and antiPF4/polyanion antibodies in Norwegian health care workers after COVID-19 vaccination. J Thromb Haemost. (2021) 19:1813-8. doi: 10.1111/jth.15352

43. Waissengrin B, Agbarya A, Safadi E, Padova H, Wolf I. Shortterm safety of the BNT162b2 mRNA COVID-19 vaccine in patients with cancer treated with immune checkpoint inhibitors. Lancet Oncol. (2021) 22:581-3. doi: 10.1016/S1470-2045(21)0 0155-8

44. CDC COVID-19 Response Team and Food and Drug Administration. Allergic reactions including anaphylaxis after receipt of the first dose of PfizerBioNTech COVID-19 vaccine - United States, December 14-23, 2020. Morb Mortal Wkly Rep. (2021) 70:46-51. doi: 10.15585/mmwr.mm7002e1

45. CDC COVID-19 Response Team and Food and Drug Administration. Allergic Reactions Including Anaphylaxis After Receipt of the First Dose of Moderna COVID-19 Vaccine - United States, December 21, 2020-January 10, 2021. Morb Mortal Wkly Rep. (2021) 70:125-9. doi: 10.15585/mmwr.mm7004e1

46. Gee J, Marquez P, Su J, Calvert GM, Liu R, Myers T, et al. First Month of COVID-19 vaccine safety monitoring - United States, December 14, 2020-January 13, 2021. Morb Mortal Wkly Rep. (2021) 70:2838. doi: 10.15585/mmwr.mm7008e3

47. Hause AM, Gee J, Johnson T, Jazwa A, Marquez P, Miller E, et al. Anxietyrelated adverse event clusters after janssen COVID-19 vaccination - Five U.S. Mass Vaccination Sites, April 2021. Morb Mortal Wkly Rep. (2021) 70:685-8. doi: 10.15585/mmwr.mm7018e3

48. Shay DK, Gee J, Su JR, Myers TR, Marquez P, Liu R, et al. Safety monitoring of the janssen (Johnson \& Johnson) COVID-19 vaccine United States, March-April 2021. Morb Mortal Wkly Rep. (2021) 70:6804. doi: 10.15585/mmwr.mm7018e2

49. Shimabukuro TT, Cole M, Su JR. Reports of anaphylaxis after receipt of mRNA COVID-19 vaccines in the US-December 14, 2020-January 18, 2021. JAMA. (2021) 325:1101-2. doi: 10.1001/jama.2021.1967

50. Skowronski DM, De Serres G. Safety and efficacy of the BNT162b2 mRNA Covid-19 vaccine. N Engl J Med. (2021) 384:1576-7. doi: 10.1056/NEJMc2036242

51. Thompson MG, Burgess JL, Naleway AL, Tyner HL, Yoon SK, Meece J, et al. Interim estimates of vaccine effectiveness of BNT162b2 and mRNA1273 COVID-19 vaccines in preventing SARS-CoV-2 infection among health care personnel, first responders, and other essential and frontline workers eight U.S. Locations, December 2020-March 2021. Morb Mortal Wkly Rep. (2021) 70:495-500. doi: 10.15585/mmwr.mm7013e3

52. Farinazzo E, Ponis G, Zelin E, Errichetti E, Stinco G, Pinzani C, et al. Cutaneous adverse reactions after m-RNA COVID-19 vaccine: early reports from North-East Italy. J Eur Acad Dermatol Venereol. (2021) 35:e54851. doi: $10.1111 /$ jdv. 17343

53. Fernández-Prada M, Rivero-Calle I, Calvache-González A, MartinónTorres F. Acute onset supraclavicular lymphadenopathy coinciding with intramuscular mRNA vaccination against COVID-19 may be related to vaccine injection technique, Spain, January and February 2021. Euro Surveill. (2021) 26:2100193. doi: 10.2807/1560-7917.ES.2021.26.10.2100193
54. Johnston MS, Galan A, Watsky KL, Little AJ. Delayed localized hypersensitivity reactions to the moderna COVID-19 vaccine: a case series. JAMA Dermatol. (2021) 157:716-20. doi: 10.1001/jamadermatol.2021.1214

55. Lee EJ, Cines DB, Gernsheimer T, Kessler C, Michel M, Tarantino MD, et al. Thrombocytopenia following Pfizer and Moderna SARS-CoV-2 vaccination. Am J Hematol. (2021) 96:534-7. doi: 10.1002/ajh.26132

56. Meylan S, Livio F, Foerster M, Genoud PJ, Marguet F, Wuerzner G. Stage III hypertension in patients after mRNAbased SARS-CoV-2 vaccination. Hypertension. (2021) 77:e567. doi: 10.1161/HYPERTENSIONAHA.121.17316

57. Román GC, Gracia F, Torres A, Palacios A, Gracia K, Harris D. Acute Transverse Myelitis (ATM):clinical review of 43 patients with COVID19-associated ATM and 3 post-vaccination ATM serious adverse events with the ChAdOx1 nCoV-19 vaccine (AZD1222). Front Immunol. (2021) 12:653786. doi: 10.3389/fimmu.2021.653786

58. Shemer A, Pras E, Hecht I. Peripheral facial nerve palsy following BNT162b2 (COVID-19) vaccination. Isr Med Assoc J. (2021) 23:143-4.

59. Global Health 5050. Gender and Sex-Disaggregated Data: Vital to Inform an Effective Response to COVID-19. (2020). Available online at: https:// globalhealth5050.org/wp-content/themes/global-health/covid/media/ISSUE \%20BRIEF\%20-\%20Sex-Disaggregated\%20Data\%20\&\%20COVID-19\%20\%20Sept\%202020.pdf (accessed June 25, 2021).

60. Vijayasingham L, Bischof E, Wolfe J. Sex-disaggregated data in COVID-19 vaccine trials. Lancet. (2021) 397:966-7. doi: 10.1016/S0140-6736(21)00384-6

61. Bischof E, Wolfe J, Klein SL. Clinical trials for COVID-19 should include sex as a variable. J Clin Invest. (2020) 130:3350-2. doi: 10.1172/JCI139306

62. Evagora-Campbell M, Borkotoky K, Sharma S, Mbuthia M. From routine data collection to policy design: sex and gender both matter in COVID-19. Lancet. (2021) 397:2447-9. doi: 10.1016/S0140-6736(21)01326-X

63. Brady E, Nielsen MW, Andersen JP, Oertelt-Prigione S. Lack of consideration of sex and gender in COVID-19 clinical studies. Nat Commun. (2021) 12:4015. doi: 10.1038/s41467-021-24265-8

64. Carcel C, Reeves M. Under-enrollment of women in stroke clinical trials: what are the causes and what should be done about it? Stroke. (2021) 52:4527. doi: 10.1161/STROKEAHA.120.033227

65. Palmer-Ross A, Ovseiko PV, Heidari S. Inadequate reporting of COVID-19 clinical studies: a renewed rationale for the Sex and Gender Equity in Research (SAGER) guidelines. BMJ Glob Health. (2021) 6:e004997. doi: 10.1136/bmjgh-2021-004997

66. Heidari S, Babor TF, De Castro P, Tort S, Curno M. Sex and gender equity in research: rationale for the SAGER guidelines and recommended use. Res Integr Peer Rev. (2016) 1:1-9. doi: 10.1186/s41073-016-0016-5

67. AusVaxSafety. COVID-19 Vaccine Safety Surveillance. (2021). Available online at: https://www.ausvaxsafety.org.au/our-work/covid-19-vaccine-safetysurveillance

68. Menni C, Klaser K, May A, Polidori L, Capdevila J, Louca P, et al. Vaccine sideeffects and SARS-CoV-2 infection after vaccination in users of the COVID Symptom Study app in the UK: a prospective observational study. Lancet Infect Dis. (2021) 21:939-49. doi: 10.1016/S1473-3099(21)00224-3

69. McCartney PR. Sex-based vaccine response in the context of COVID-19. J Obstet Gynecol Neonatal Nurs J. (2020) 49:4058. doi: 10.1016/j.jogn.2020.08.001

70. Himmelstein MS, Sanchez DT. Masculinity impediments: Internalized masculinity contributes to healthcare avoidance in men and women. J Health Psychol. (2016) 21:1283-92. doi: 10.1177/1359105314551623

71. Thompson AE, Anisimowicz Y, Miedema B, Hogg W, Wodchis WP, AubreyBassler K. The influence of gender and other patient characteristics on health care-seeking behaviour: a QUALICOPC study. BMC Fam Pract. (2016) 17:38. doi: 10.1186/s12875-016-0440-0

72. Feletto M, Sharkey A. The influence of gender on immunisation: using an ecological framework to examine intersecting inequities and pathways to change. BMJ Glob Health. (2019) 4:e001711. doi: 10.1136/bmjgh-2019001711

73. Sherman SM, Smith LE, Sim J, Amlôt R, Cutts M, Dasch H, et al. COVID-19 vaccination intention in the UK: results from the COVID-19 vaccination acceptability study (CoVAccS), a nationally representative cross-sectional survey. Hum Vaccin Immunother. (2021) 17:1612-21. doi: 10.1080/21645515.2020.1846397 
74. Loomba S, de Figueiredo A, Piatek SJ, de Graaf K, Larson HJ. Measuring the impact of COVID-19 vaccine misinformation on vaccination intent in the UK and USA. Nat Hum Behav. (2021) 5:337-48. doi: 10.1038/s41562-021-01056-1

75. Brewer NT, Chapman GB, Rothman AJ, Leask J, Kempe A. Increasing vaccination: putting psychological science into action. Psychol Sci Public Interest. (2017) 18:149-207. doi: 10.1177/15291006187 60521

76. Bish A, Yardley L, Nicoll A, Michie S. Factors associated with uptake of vaccination against pandemic influenza: a systematic review. Vaccine. (2011) 29:6472-84. doi: 10.1016/j.vaccine.2011.06.107

77. Solis Arce J, Warren S, Meriggi N, Scacco A, McMurry N, Voors $\mathrm{M}$, et al. COVID-19 vaccine acceptance and hesitancy in low and middle income countries, and implications for messaging. Nat Med. (2021) 27:1385-94. doi: 10.1101/2021.03.11. 21253419

78. Zintel S, Flock C, Arbogast AL, Forster A, von Wagner C, Sieverding M. Gender Differences in the Intention to Get Vaccinated against COVID-19 - A Systematic Review and Meta-Analysis (March 12, 2021). Available online at: https://papers.ssrn.com/sol3/papers.cfm?abstract_id=3803323 (accessed June 25, 2021)

79. Vassallo A, Womersley K, Norton R, Sheel M. Pregnant women's appetite for risk. Lancet Global Health. (2021) 9:e593. doi: 10.1016/S2214-109X(21)00044-9

80. Villarreal A. 'No data' linking Covid vaccines to menstrual changes, US Experts Say. The Guardian (2021). Available online at: https://www.theguardian.com/ world/2021/apr/23/covid-vaccines-periods-menstruation-changes-dataexperts (accessed June 2021).

81. Robinson OSR. Covid Vaccine: Period Changes Could be a Short-Term Side Effect. BBC (2021). Available online at: https://www.bbc.com/news/health56901353 (accessed June 2021).

82. Schraer R. Covid: Claims Vaccinations Harm Fertility Unfounded: BBC (2021). Available online at: https://www.bbc.com/news/health-56012529 (accessed June 2021).

83. Wise M. Could the COVID Vaccines Affect Your Period? We don't Know Yet - but There's no Cause for Concern. The Conversation. (2021). Available online at: https://theconversation.com/could-the-covid-vaccines-affectyour-period-we-dont-know-yet-but-theres-no-cause-for-concern- 159912 (accessed June 2021).

84. Ahmed SB, Dumanski SM. Sex, gender and COVID-19: a call to action. Can J Public Health. (2020) 111:980-3. doi: 10.17269/s41997-020-00417-z

85. Woodward M. Rationale and tutorial for analysing and reporting sex differences in cardiovascular associations. Heart. (2019) 105:1701-8. doi: 10.1136/heartjnl-2019-315299

86. World Health Organization. Strategy for Integrating Gender Analysis and Actions Into the Work of WHO. Geneva: WHO Department of Gender, Women and Health (2008).

87. Heidari S, Ahumada C, Kurbanova Z. Towards the real-time inclusion of sexand age-disaggregated data in pandemic responses. BMJ Glob Health. (2020) 5:e003848. doi: 10.1136/bmjgh-2020-003848

Conflict of Interest: MW is a consultant to Amgen, Kyowa Kirin, and Freeline.

The remaining authors declare that the research was conducted in the absence of any commercial or financial relationships that could be construed as a potential conflict of interest.

Publisher's Note: All claims expressed in this article are solely those of the authors and do not necessarily represent those of their affiliated organizations, or those of the publisher, the editors and the reviewers. Any product that may be evaluated in this article, or claim that may be made by its manufacturer, is not guaranteed or endorsed by the publisher.

Copyright (c) 2021 Vassallo, Shajahan, Harris, Hallam, Hockham, Womersley, Woodward and Sheel. This is an open-access article distributed under the terms of the Creative Commons Attribution License (CC BY). The use, distribution or reproduction in other forums is permitted, provided the original author(s) and the copyright owner(s) are credited and that the original publication in this journal is cited, in accordance with accepted academic practice. No use, distribution or reproduction is permitted which does not comply with these terms. 